\title{
Structure of the murine Thy-1 gene
}

\author{
Vincent Giguère, Ken-Ichi Isobe ${ }^{1}$ and Frank Grosveld \\ Laboratory of Gene Structure and Expression, National Institute for Medical \\ Research, The Ridgeway, Mill Hill, London NW7 1AA, and ${ }^{1}$ Department \\ of Zoology, University College London, Gower Street, London WC1E 6BT, \\ UK \\ Communicated by A. F. Williams
}

We have cloned the murine Thy-1.1 (AKR) and Thy-1.2 $(\mathrm{Balb} / \mathrm{c})$ genes. The complete exon/intron structure and the nucleotide sequence of the Thy-1.2 gene was determined. The gene contains four exons and three intervening sequences. The complete transcriptional unit gives rise to a tissue and developmental stage-specific mRNA of $1850 \mathrm{bp}$. The $5^{\prime}$ end of the gene has multiple initiation sites and a non-TATA box promoter. The $3^{\prime}$ end shows a single polyadenylation site after a very long untranslated region.

Key words: Thy-1/gene structure

\section{Introduction}

The Thy- 1 antigen is a small glycoprotein found in large amounts at the cell surface of brain cells and rodent thymocytes, and in lesser quantity on a number of other tissues (Campbell et al., 1981). In many of these tissues, the level of expression of Thy-1 has been shown to vary during differentiation (Owen and Raff, 1970; Schachner and Hämmerling, 1974; Bash and Berman, 1982). In the mouse, Thy-1 occurs in two allotypic forms referred to as Thy-1.1 (AKR) and Thy-1.2 (Balb/c and others) (Reif and Allen, 1964) encoded by a gene that maps on chromosome 9 (Blankenhorn and Douglas, 1972). Structural and sequence homologies between the Thy-1 glycoprotein and immunoglobulin (Ig) domains and other members of the Ig superfamily (Cohen et al., 1981; McNicholas et al., 1982; Mostov et al., 1984; Clark et al., 1985) have led to the suggestion that Thy-1 may represent the primordial Ig domain (Williams and Gagnon, 1982) lending support to the argument that the immune system may have evolved from the nervous system. Thus, the study of the Thy- 1 gene is interesting, both from the point of view of its structure and the mechanisms which control its tissue-specific expression.

We describe here the isolation of the mouse Thy-1.1 and Thy-1.2 genes. We present the complete exon/intron organization of the mouse Thy-1.2 gene, including the structure of its 5 ' end exon and flanking promoter region. The results show that the genomic organization of the Thy-1 gene is similar to that of the other members of the Ig superfamily. The exceptions are the $5^{\prime}$ end exon and the promoter region which are different from those of the Ig superfamily and from the majority of previously studied eukaryotic genes.

\section{Results}

Isolation of the genomic fragment encoding the mouse Thy-1.2 gene

To isolate the mouse Thy-1.2 gene, we used a rat Thy-1 cDNA (Moriuchi et al., 1983) to screen a mouse cosmid library con- structed from a partial MboI digestion of WEHI 3B cell line DNA ligated to the vector pTCF (Grosveld et al., 1982b). Approximately $4 \times 10^{5}$ recombinants were screened, and we isolated one clone that hybridized strongly to the rat Thy- 1 cDNA probe. This clone, pMT1, contains a $40 \mathrm{~kb}$ insert which was mapped using conventional restriction enzyme double digests. Only the EcoRI restriction map is shown in Figure 1. Hybridization with the rat cDNA showed that the mouse Thy-1.2 gene was located on a single 8.2-kb EcoRI fragment. To facilitate sequence analysis and study of the expression of the mouse Thy-1.2 gene, the $8.2-\mathrm{kb}$ EcoRI fragment was subcloned into the expression vector $\mathrm{pBSV}$ (Grosveld et al., 1982a), giving rise to the plasmid pBST1, to allow expression from the Thy- 1 promoter in the presence of an SV40 enhancer.

We then established that the 8.2-kb EcoRI genomic fragment contains the entire functional Thy-1.2 gene by sequence analysis (see below) and DNA-mediated gene transfer. Human HeLa cells were transfected with the subclone pBST1, collected 2 days later and assayed for the presence of the Thy-1.2 antigen on the cell surface using a monoclonal antibody against Thy-1.2 (Lake, 1979). The HeLa cells show $30 \%$ of the binding seen with the positive control BW5174, the T-cell lymphoma cell line (Goldsby et al., 1977, data not shown). The results indicate that the HeLa cells express the mouse Thy-1.2 antigen very efficiently on the cell surface when transformed with pBST1. If we assume a transformation efficiency of $20-40 \%$ (Banerji et al., 1981), the transformed HeLa cells actually express the Thy-1 antigen at the same level as the positive control BW5174. Since it is known that the introduced gene is usually transcribed from its own promoter in this system (Banerji et al., 1981), it is very likely that the 8.2-kb EcoRI fragment contains the entire transcriptional unit of the mouse Thy-1.2 gene.

Nucleotide sequence and complete exon/intron structure of the mouse Thy-1.2 gene.

To study the intron/exon organization of the Thy-1.2 gene we obtained additional cDNA clones from a rat thymocyte cDNA library (Clark et al., 1985) because the pT64 cDNA (Moriuchi et al., 1983) represents only part of the Thy-1 mRNA transcript. One of the new cDNA clones, pT2, with the longest insert (1600 bp) was selected for complete sequence analysis. At the same time we isolated all the mouse Thy-1.2 genomic fragments from pBST1 which hybridized to pT2. The strategy used to sequence these fragments, which contain the entire transcriptional unit as well as $600 \mathrm{bp}$ of $5^{\prime}$-flanking sequences, is shown in Figure 1. Comparison of the genomic pBST1 sequence with the sequence obtained from the pT2 cDNA permitted the precise identification of the exon/intron boundaries; all the splice sites are of the type 1 as in the other genes of the Ig superfamily (Sharp, 1981) and in agreement with the consensus sequence at exon/intron boundaries (Benoist et al., 1980). The 3' end of the pT2 sequence agrees with a polyadenylation signal at position 6122 (Figure 2) and represents the $3^{\prime}$ end of the mRNA (see below). Lastly, in contrast to the previously reported structure of the gene (Evans 


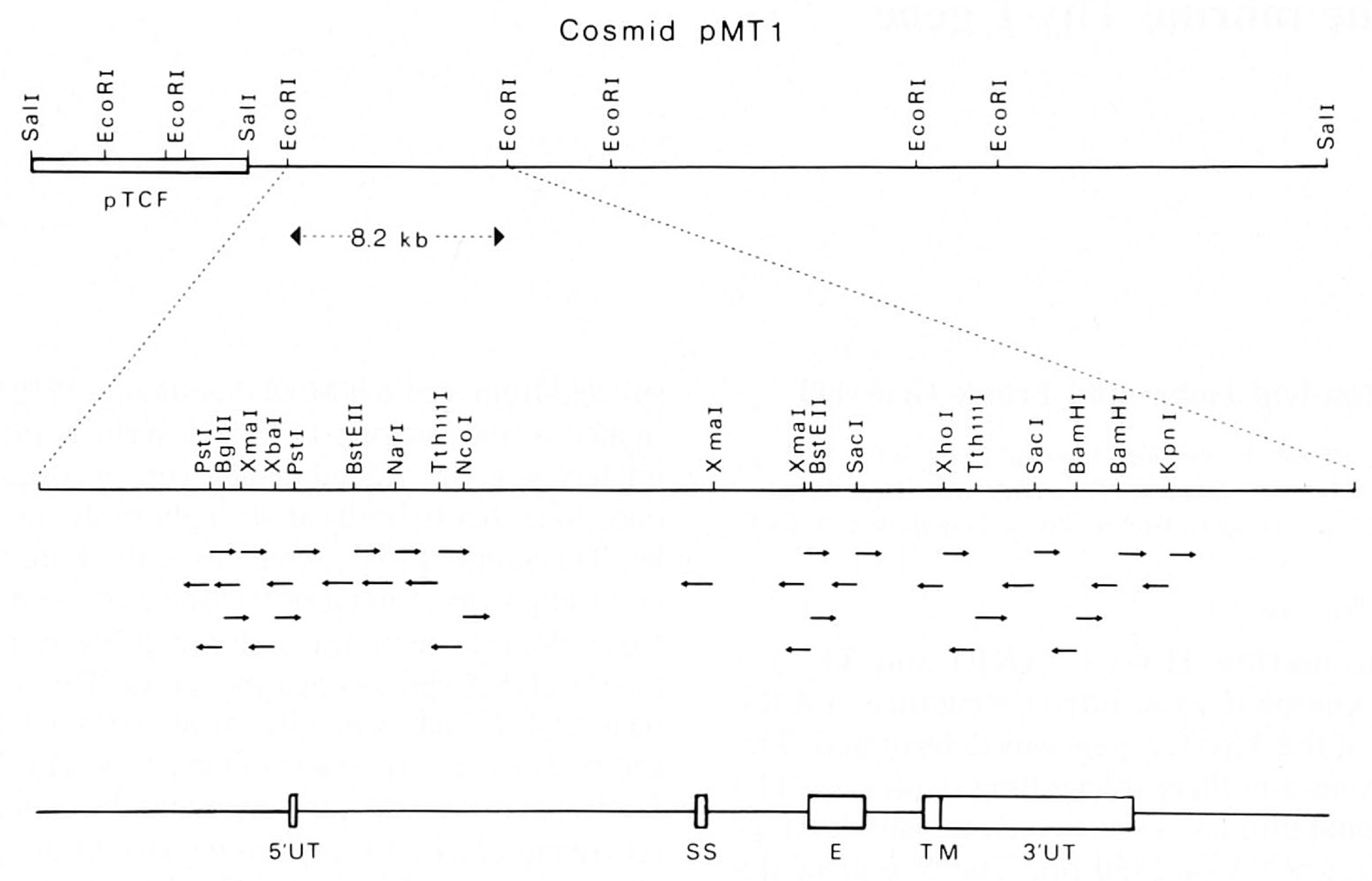

Fig. 1. Organization and sequencing strategy of the genomic clone pMT1 containing the Balb/c mouse Thy-1.2 gene. Only the restriction sites used for sequencing are marked. The arrows indicate the direction and extent of the fragments sequenced. Exons are depicted as boxes, introns and flanking regions as lines. 5'UT, 5'-untranslated region; SS, signal sequence peptide; E, extracellular domain; TM, transmembrane-like domain; 3'UT, 3'-untranslated region.

et al., 1984; Seki et al., 1985), the first 27 bp at the $5^{\prime}$ end of the pT2 sequence indicate the existence of at least one more exon $2.6 \mathrm{~kb}$ upstream to bring the total number to four exons (see $5^{\prime}$ end analysis below).

The exon/intron organization of the mouse Thy-1.2 gene corresponds approximatively to the functional domains of the protein, and is similar to the other members of the Ig superfamily (Hood et al., 1985). The exception is the $5^{\prime}$-untranslated region of the mRNA which is encoded by two exons, exon 1 and part of exon 2 which also contains the signal peptide. Exon 3 codes for the external domain of the protein. Exon 4 codes for a transmembrane-like domain and an unusually long 3 '-untranslated region.

\section{3' End analysis of the mouse Thy-1.2 gene}

On the basis of their first analysis of a rat Thy-1 cDNA, Moriuchi et al. (1983) proposed that the Thy-1 gene could be subject to differential polyadenylation. For a complete $S 1$ analysis of the $3^{\prime}$ end of the Thy-1 mRNA, we used two different probes derived from genomic DNA. The first one (Figure 3B) is a 344-bp Hinfl fragment extending from nucleotide 5932 to 6276 (see Figure 2 for numbering) and covers the polyadenylation signal AATAAA located at position 6122. The second is an exon/intron border probe which corresponds to an 805-bp fragment extending from a BstEII site located at position 4255 in the third exon to an ApaI site situated in the fourth exon (Figure 3C), and covers the proposed polyadenylation signal AATAAA at position 4543 (Moriuchi et al., 1983). These probes were hybridized to total RNA from BW5147 cells, the heteroduplexes were digested with $\mathrm{S} 1$ nuclease, and the length of the protected fragments was determined on a denaturing $5 \%$ polyacrylamide gel. As shown on Figure 3A, the Hinfl probe generates two protected fragments of $\sim 210$ nucleotides, which would situate the polyadenylation site $\sim 12-15$ bp downstream the polyadenylation signal at position 6122 . This is in agreement with the sequence of the rat Thy-1 cDNA clone pT2 (Figure 4B), which puts the polyadenylation site $12 \mathrm{bp}$ from the sequence AATAAA.
A single protected fragment of 310 nucleotides in length is observed when the BstEII/ApaI probe is used (Figure 3A, lane 4), which corresponds exactly to the border between exon 3 and intron 4 . We did not detect any bands at 300 nucleotides, corresponding to the poly (A) site in the third exon as suggested by Moriuchi et al. (1983). Our results are in agreement with the finding that only one species of mRNA of $\sim 1850$ nucleotides is detected with a mouse Thy-1 probe on a Northern blot of total RNA from BW5147 cells (Figure 5) or brain and thymus mRNA (not shown). Interestingly, the same mRNA is developmentally regulated in F9 embryonal carcinoma cells. Figure 5 (lanes 2 and 3 ) shows that the Thy-1 mRNA is undetectable before differentiation but switched on after differentiation of the F9 cells with retinoic acid (Hogan et al., 1981). Consequently it appears that the same transcriptional unit is utilized in different tissues and in early development since it is active in F9 cells, which are thought to represent early embryonic cell types giving rise to parietal and visceral endoderm (Hogan et al., 1981).

\section{5' End analysis of the mouse Thy-1.2 gene}

The sequence analysis revealed that the only sequence which matches the first 27 bp of the rat Thy- 1 cDNA clone pT2, is located $2.6 \mathrm{~kb}$ upstream from the exon containing the initiation codon methionine (Figures 2 and 4A). In order to define the precise 5 ' end of the mouse Thy-1 mRNA, we have used a 5'-labelled 31-nucleotide Hinfl/HhaI fragment which is part of the putative first exon as a primer for extension. The result of this analysis (Figure 6A) shows three major extension products; the longest corresponding to an A residue at position 594 in Figure 2, the two other bands correspond to a $\mathrm{G}$ and an $\mathrm{A}$ residue at position 595 and 597, respectively. Overexposure of the film to the gel reveals additional weak bands corresponding to $5^{\prime}$ end positions indicted by open triangles in Figure 2. To exclude the possibility that the extended products seen by primer extension correspond to strong stops for the reverse transcriptase, we confirmed the $5^{\prime}$ end with an 51 nuclease protection experiment using total RNA from BW5147 cells. A 520-nucleotide PstI fragment 

CAGATCTCCAGCCAAGAGGCAAAGGAATGGOGGAAGCTGC AGGGCCTCCCTCTGGTT ATCCAGGCTTCTG AAGG TTCAAGCAAAGAAAGGGTTACAACCT TAAAAGGAGAGCGTCCCGGGGTATGGGTAGAAGACTGCTCCACCCCGACCCCCAGGGTCCCT AACCGTCTTTTCCTTGGGCGAGTCAGCCCAATCACAGG ACTGAGAGTGCCTCTTTAGTAGCAGCAAGCCACTTCGGACACCCAAATGGAACACCTCCAGTCAGCCCTCGCCGACCACCCCACCCCCTCCATCCTTTTC CCTCAGCCTCCGATTGGCTGAATCTAGAGTCCCTCCCTGCTCCCCCCTCTCTCCCCACCCCTGGTGAAAACTGCGGGCTTCAGCGCTGGGTGCAGCAACT GGAGGCGTTGGCGCACT AGGAGGAGGCTGCAGCT AGGGGAGTCT AGETGAGAGCAGGCCGACGGGAGGGACCCGCACATGCAAGGACCGCCGCAGGGCGG GATGCAAGCCTTCCCCAGCT ACAGTTTTGGGAAGGAT ACTAAGGCGCTCCT AT ATGGGGGCGCGGGAACTTGGGGAAAGAAGGTGCTCCCAAGTCGAGG TGGGAGAGGAAGGCAGTGCGGGG TCACGGGCT TTCT CCCTGCT AACGGACGCTTTCGAAGAGTGGG TGCCGG AGGAGAACCATGAGGAAGGACATCAAGG ACATCAAGGACAGCCTTTGGTCCCCAAGCTCAAATCGCTTT AGTGGTGCGA ATAGAGGGAGGAGG TGGGTGGCAAACTGGAGGGAGCCCCCAACGGGTGA CCTCGTGGCTGGCTGGGTGCGGGG TAGT AGGT AAGAAAACCGCAATGTTGCGGGAGGGGACTGGGTGGCAGGCGCGGGGAGGGGAAAGCT AGAAAGGAT GCGAGGGAGCGG AGGGGGGAGGGAGCGGGGGAAT CT CAACTGGT AGAGGAAAGTT AAAATGAGG AAAT AGCATCAGGG TGGGGTT AGCCAAGCCGGGCCT CAGGGAAAGGGCGCAAAGTTTG TCTGGGTG TGGGCTTAGG TGGGCTGGGTATGAGATTCGGGGCGCCGAAAACACTGCTGCGCCTCTGCCAAATCACGCT ACCCCTGTATCT AGTTCTGCT AGGCTTCTCCAGCCCCAGCCCCAATTCTTTTCTCAGTGTCCCCTTCCCTCCCCTGAATCTCAAGCCCACACTCCCTCCT CCATAACCCACTGTTATCAAATCT AAGTCATTTGCCACCCAACAACCATCAGGAGGCGGAAGCAGACGGGAGGAGTTTGAGATCAACTTGGGCT ACATCA CGAGTTCCAAGCTCACCAAGGCTTCTT AAGGAGACCTTGTCTCT AAAATT AATT AATT AATT AATT AAT AGTCCCCTTTCTCTGCCACAGAACCTTGGGA TCTGGCTCCTGGTCGCAGCTCCCCCCACCCCAGGCTGACATTCACTGCCATAGCCCATCCGGAAATCCTAGTCT ATTTCCCCATGGATCTTGAACTGCAG AGAGA ATGGCAGAGTGGCCCGCCCTG TGCAAAGGATGTTCCT AGCCT AGGTGGAGCTCGCGAACTCGCAGACTG TGCCTCTCTTGGGCAAGGACAGGCT A GACAGCCTGCCGGTGTGTTGAGCTAGGGCACTGTGGGG..................... $1.4 \mathrm{~kb}$ $-19$ ... CACCTGTCCTACCAGCTGGTTGACCTGTAGCTTTCCCCACCACAGAATCCAAGTCGGAACTCTTGGCACCATGAACCCAGCCATCAGCGTCGCTCTC $-10$

....... ACCAAT ACCTCAAGGGCGAGTGCAAACCCCACATCCCCCCAGCTCAAGTCT ACTTTTTCCTGCAGG TGGGAGGCCCCGGGTCTGTGTCTCCC $-1 \quad 1$

alLeuGlnValSerArgGlyGlnLy 8V 1 IThrSerLeuThrAlaCy вLeuVglAsnG InABnLeuArg Leu CAAATTCAGAGAAGGCACTGCTGTGCCAGTCTTGCAGGTGTCCCGAGGGAGAAGGTGACCAGCCTGACAGCCTGCCTGGTGAACCAAAACCTTCGCCTG 20 30 40

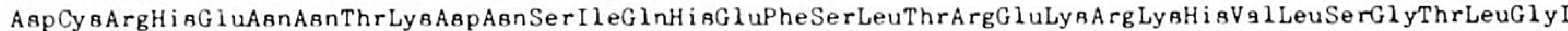
GACTGCCGCCATGAGA AT AACACCAAGGAT AACTCCATCCAGCATGAGTTCAGCCTGACCCGAGAGAAGAGGAAGCACGTGCTCTCAGGCACCCTTGGGA $60 \quad 70 \quad 80$

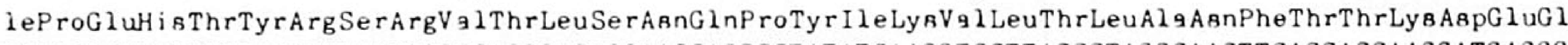
TACCOGAGCACACGT ACCGCTCCCGCGTCACCCT CTCCAACCAGCCCT AT AT CAAGGTCCT T ACCCT AGCCAACTTCACCACCAAGGATGAGGGCGACT A

rPheCy BGluLeuGinValSerGIyAlaABnProMetSerSerAsnLy BSerILeSerValTyrArgA 100 CTTTTGTGAGCTTCAAGTCT CGGGCGCGATCCCATGAGCTCCAAT AAAAGTATCAGTGTGTAT AGAGGTGAGACTGGTTCCCAGAGAGATAAAATGTCT AGGTT AGCT AGGCTGGGG TAGCCAAT AAAAAAAAAAAAAAAACGGGCACCTCCATT ACCCTTCCCCT AACTGCTGGTCTCCTGGGAAACTGCTGCTGTCT ATGTGAGTGGGGAAGATTAGGGG ......... About $0.16 \mathrm{~kb} \ldots \ldots \ldots . .$. CCGCCTGAGTCCTGATCTCCCCTCCGCCCGGCACCCCTTCTCT A 110

120

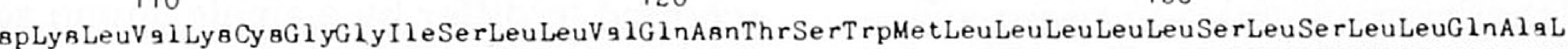
TCCACAGACAAGCTGGTCAAGTGTGGCGGCAT AAGCCTGCTGGTTCAGAACACATCCTGGATGCTGCTGCTGCTGCTTTCCCTCTCCCTCCTCCAAGCCC $140 \quad 143$

eUAspheIleSerLeuTEK

TAGACTTCATTTCTCTGTGACTGG TTGGGCCCAAGG AGAAACAGGAGCCCTCGAGGTCCTTCCTCTGCAGAGGTCTTGCTTCTCCCGG TCAGCTGACTCC CT CCCCAAGTCCTTCAAAT ATCTCAGAACATGGGGAGAAACGGGGACCTTGTCCCTCCT AAGGAACCCCAGTGCTGCATGCCATCATCCCCCCCACCCTC GCCCCCACCCCCGCCACTTCTCCCTCCATGCATACCACTAGCTG TCATTTTGTACTCTGTATTT ATTCTAGGGCTGCTTCTGATTATTTAGTTTGTTCTT TCCTTGGAGACCTG TT AGA ACAT AAGGGCGT ATGGTGGGT AGGGGAGGCAGG AT ATCAGTCCCTGGGGCGAGTTCCTCCCTGCCAACCAAGCCAGATGCC TGAAAGAGAT ATGGATGAGGGAAGTTGGACTGTGCCTGCACCTGGT ACAGTCACACTCTGTTGAAAGAATCATCGGGGAGGGGGGGGGGTCAAGAGGGG AGAGCTCTGCTGAGTCTTTG TGGACCATCCAATGAGGATGAGGGCT TAGATTCT ACCAGG TCATTCTCAGCCACCACACACAAGCGCTCTGCCATCACTG AAGAAGCCCCCT AGGGCCTTGGGCCAGGGCACACTCAGT AAAGA TGCAGG TTCAGTCAGGGATG ATGGGGAAAGGGG TAGG AGGTGGGGGAGGATCAC CCCCTCCT CT AAAACACGAGCCTGCTGCCTCCAAAGGCCTCTGCCTGTAGTGAGGGTGGCAGAAGA AGACAAGGAGCCAGAACTCTGACTCCAGGATCT A AGTCCGTGCAGGAAGGGGATCCT AGAACCATCTGGTTGGACCCGGCTT ACCAAGGGAGAGCCTTT ATTCTTCTTTTCCCTTGCCCCTCTGTGCCAGCCCC TCTTGCTGTCCCTGATCCCCCAGACAGCGAGAGTCTTGCAACCTGCCTCT TCCAAGACCTCCT AATCTCAGGGGCAGGCGGTGGAGTGAGATCCGGCGTG CACACTTTTTGGAAGATAGCTTTCCCAAGGATCCTCTCCCCCACTGGCAGCT CTGCCTGTCCCATCACCATGTG TAATACCACCACTGCT ACAGCGTCTC ACCGAGGAAAGAAAACTGCACAATAAAACCAAGCCTCTGGAGTG TGTCCTGGTG TCTGTCTCTTCTGTGTCCTGGCGTCTGTCTCTTCTG TGTTCTTCCA AGGTCAGA AACAAAAACCACACACTTCAACCT AGATGGCTCGGCTGAGCACTTCTGTGTGCAGAAGGTCCAACCGACTCTGGGTACCCGGCCCTCCCT AT TCCCTTGCCTCCTGTCTCCCGCTTTTTATAGCTCCCTATGCTGGGCTTCTCTGGAGAGTGAAATCTTTGCCCAAATCAATGCGCATTCTCTCTGCTGAGT CATCTGGCGACAGCAGTTTGAGTTCACCCGCCAAACACATGGGCCCAGCT ATGTAG

Fig. 2. DNA sequence of the mouse Thy-1.2 gene. The amino acid sequence is shown above the DNA sequence. The splice signal dinucleotides GT and AG and the polyadenylation signal AATAAA are underlined. The location of the mRNA cap sites as determined by S1 nuclease and primer extension analysis are indicated by circles and triangles, respectively. Open symbols indicate weak bands on the autoradiographs. 
(Figure 6C) was 5' end-labelled with polynucleotide kinase, hybridized and treated with $\mathrm{S} 1$ nuclease. In agreement with the primer extension, more than one band is observed on the gel (Figure 6B). The longest protected fragment of 36 nucleotides corresponds to a $\mathrm{G}$ residue at position 592 while other bands correspond to the same positions as mapped by the primer exten-

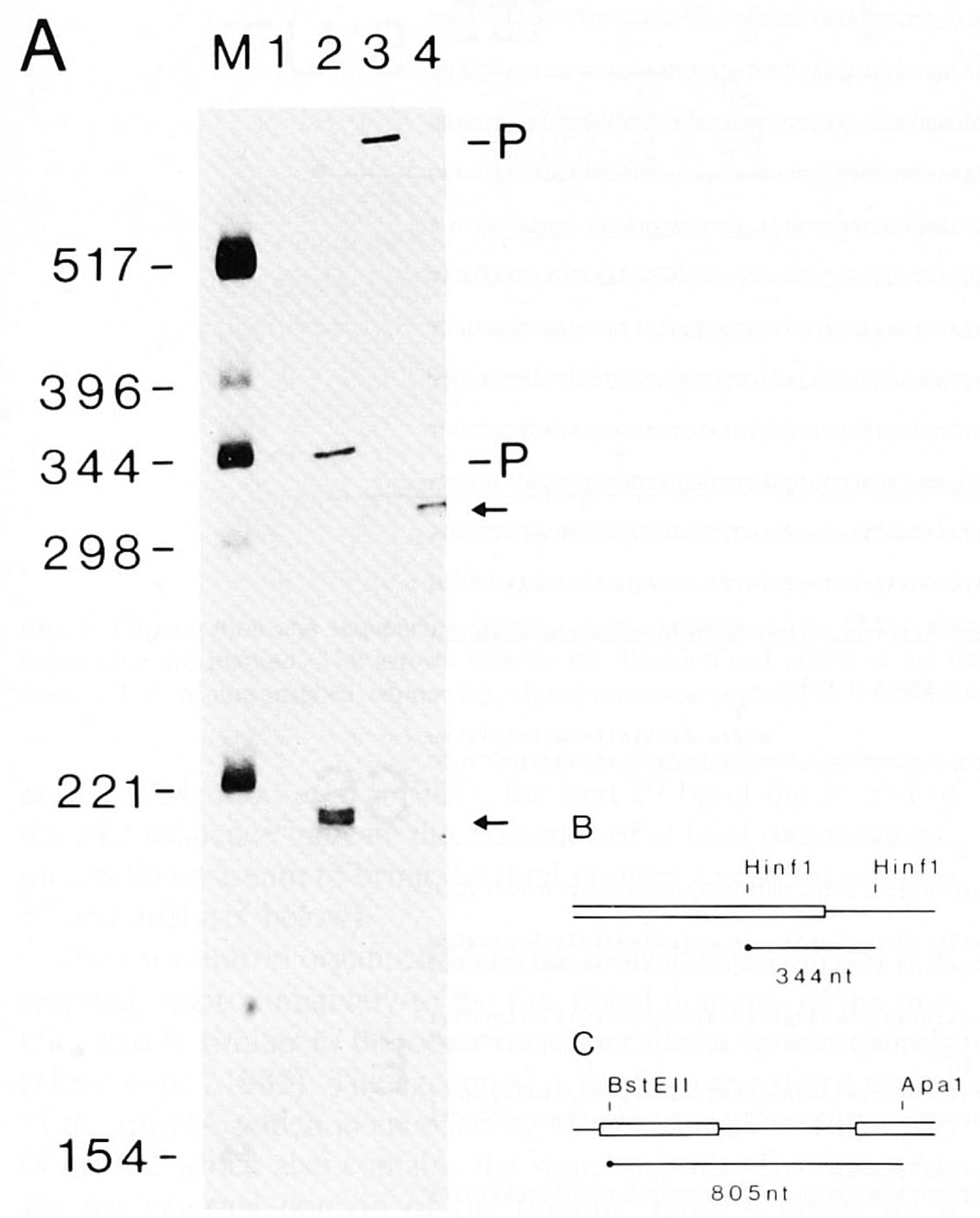

Fig. 3. Mapping the polyadenylation site at the $3^{\prime}$ end of the Thy-1 mRNA. Total BW5147 cells RNA (25 $\mu \mathrm{g}$, lanes 2 and 4) or yeast tRNA $(10 \mu \mathrm{g}$, lanes 1 and 3) was annealed to $3^{\prime}$ end-labelled probes and RNA/DNA hybrids were digested with S1 nuclease (Weaver and Weissman, 1979). The autoradiogram is shown in panel A. The Hinfl $3^{\prime}$ end probe shown on panel $\mathbf{B}$ was used in lanes $\mathbf{1}$ and $\mathbf{2}$, the BstEII/ApaI exon 3/intron 4 junction probe shown on panel $C$ was used in lanes 3 and $\mathbf{4}$. The markers were pBR322 x Hinfl. P indicates the positions of the input probes and arrows the positions of the protected fragments. sion analysis. It should be noted that the characteristic TATA (and CAAT) box usually present in eukaryotic promoters are absent in the region upstream of these putative cap sites, instead, it contains an A-rich sequence at -26 and several CG-rich areas as found in other non-TATA box promoters, in particular, the hexanucleotide CCTTGGTG at -34 which is also found in the mouse dihydrofolate reductase (DHFR) promoter (Crouse et al., 1982).

To show that these sequences can initiate transcription, the 520-bp Pst I fragment containing the Thy-1 gene 5' end was cloned in front of the chloramphenicol transferase (CAT) gene with HindIII linkers to yield the plasmid $\mathrm{pBr}$.T1.CAT. In addition to $\mathrm{pBr}$.T1.CAT, we made a further construct (pBr.T1.SV.CAT) by inserting the SV40 enhancer element at the $3^{\prime}$ side of the CAT gene (Figure 7). Both of these constructs and the control plasmids pSV2CAT and pSV1CAT (Gorman et al., 1982) were introduced into L-cells by calcium phosphate precipitation (Wigler et al., 1979) and in BW5147 cells (not shown) with DEAE dextran (Deans et al., 1984). After $60 \mathrm{~h}$ the cells were harvested and analyzed for CAT activity (Gorman et al., 1982). The L-cell result (Figure 8) shows that the Thy- 1 promoter $(\mathrm{pBr}$.T1.CAT) is comparable with the SV40 promoter (pSV1.CAT) without enhancer (lanes 2 and 4, Figure 8). The Thy-1 promoter is stimulated to efficient expression by the insertion of the SV40 enhancer $3^{\prime}$ to the CAT gene (pBr.T1.SV.CAT, lane 3), and is comparable with the SV40 promoter plus enhancer (pSV2.CAT, lane 1). A similar result is obtained with the BW5174 cells at a much lower efficiency due to a very inefficient rate of transformation. The stimulated transcription with the SV40 enhancer is in agreement with the observed efficient expression of the whole Thy-1 gene (see above) in the expression plasmid pBSV which also cotains an SV40 enhancer (Grosveld et al., 1982a).

\section{Comparison of the mouse Thy-1.1 gene and Thy-1.2 gene}

Southern blot analysis of several different mouse strains showed restriction fragment length polymorphisms (RFLPs) for the Thy-1 gene, most notably between the Thy-1.2 (Balb/c) gene and the Thy-1.1 (AKR/j) gene. The product of these genes has been reported to differ by a single amino acid at position 89 (Figure 2; Cotmore et al., 1981; Williams and Gagnon, 1982). However, of the fragments generated by treatment with restriction enzymes tested, only the EcoRI fragments were very similar (data not shown). To analyze this in more detail, the Thy-1.1 gene from $\mathrm{AKR} / \mathrm{j}$ mice was cloned as an 8.2-kb EcoRI fragment in $\lambda$ phage

\begin{abstract}
A
Mouse Thy-1.2 gene Rat Thy-1 cDNA
\end{abstract}

Fig. 4. Comparison of the mouse Thy-1.2 genomic DNA sequence with the rat Thy-1 cDNA clone pT2. (A) exon I of the mouse Thy-1.2 gene as compared with the 5' end of the rat cDNA. (B) sequences polyadenylation signal at the $3^{\prime}$ end of the cDNA and the mouse Thy-1.2 gene. (|) indicates nucleotide differences. 


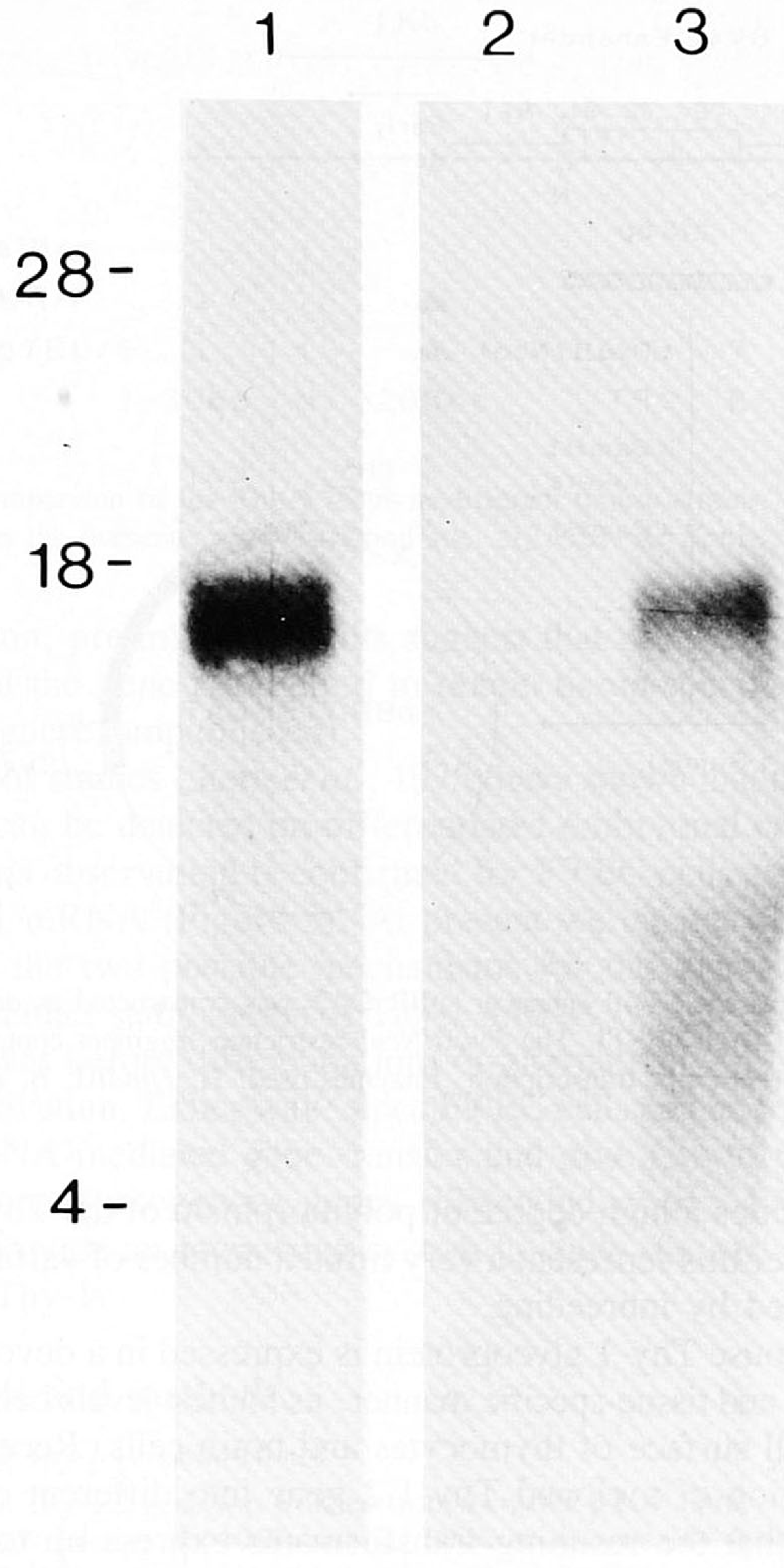

Fig. 5. Northern blot analysis of the Thy-1 mRNA. Lane 1, $25 \mu \mathrm{g}$ total BW5147 cell RNA. Lanes 2 and 3, $10 \mu \mathrm{g}$ poly(A) ${ }^{+}$RNA obtained from undifferentiated and differentiated F9 cells, respectively. The probe used to hybridize these blots is the BstEII/ApaI restriction fragment shown in Figure 3 , panel C. Size markers are mouse ribosomal $28 \mathrm{~S}$ and $18 \mathrm{~S}$ RNA and $4 \mathrm{~S}$ tRNA.

641. (Murray et al., 1977). Twelve positive in $10^{5}$ plaques were identified and the 8.2-kb insert was subcloned in the expresison vector $\mathrm{pBSV}$. As expected, sequence analysis showed a single base pair substitution $(A \rightarrow G)$ at the amino acid 89 position changing the Gln to Arg, to account for the two different allotypes of the murine Thy- 1 antigen. In addition to this, both sequence and restriction enzyme mapping showed additional differences between the genes (Figure 9); a 30-bp and 200-bp deletion/insertion at $\sim 1100$ bp and 800 bp upstream from the CAP site, respectively, a 160-bp insertion/deletion in the first intron, a 13-bp insertion/deletion in the third intron and, finally, an 11-bp insertion in the last exon. Moreover, a number of restriction sites (KpnI, NcoI, XbaI) have changed in the first intron. All of these changes have been confirmed by genomic blotting data (not shown). Inerestingly, when the Thy- 1.1 gene from $A K R / j$ is compared with the partial sequence of the Thy-1.2 gene from C57.BL/6 (Seki et al., 1985), the homology is higher than the homology found between the Thy-1.2 gene from C57.BL/6 and the Thy-1.2 gene from Balb/c.

\section{Discussion}

We have cloned the genes encoding the two allotypic forms of the mouse Thy- 1 antigen and the structure of the mouse Thy-1.2
A

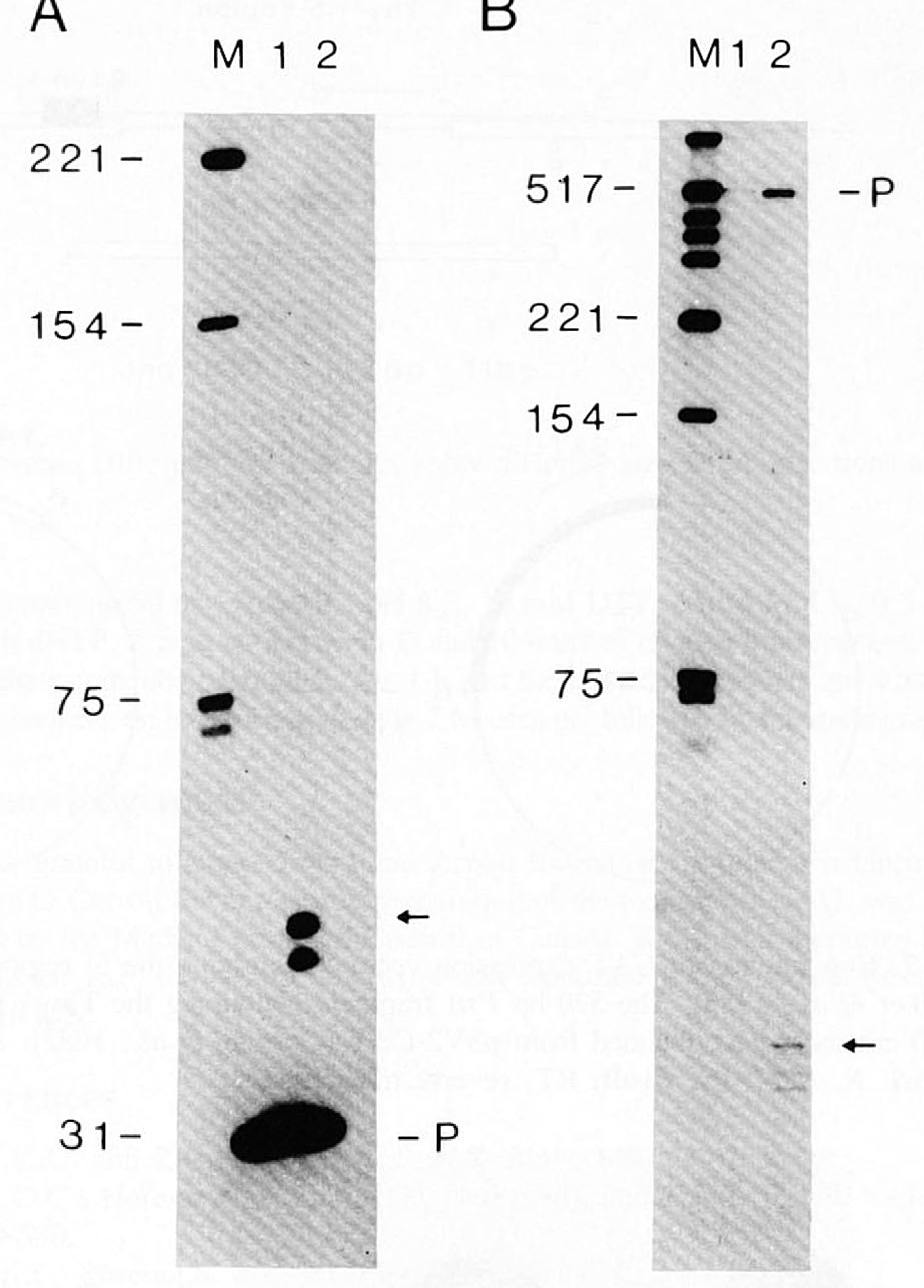

C

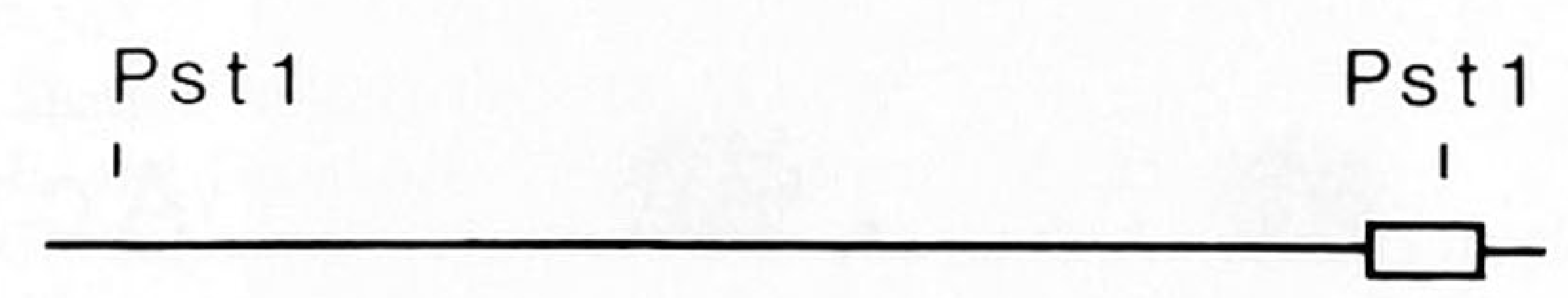

$520 \mathrm{nt}$

Fig. 6. Mapping the sites of transcription initiation by primer extension and S1 nuclease analysis. Panel A shows the result of the primer extension analysis using a 31-nucleotide primer complementary to the position $612-643$ (see Figure 2) and $25 \mu \mathrm{g}$ of total RNA from BW5147 cells (lane 2) or $10 \mu$ g yeast tRNA (lane 1). Panel B shows the result of the S1 nuclease analysis. Total RNA from BW5147 cells (25 $\mu \mathrm{g}$, lane 2) or yeast tRNA $\left(10 \mu \mathrm{g}\right.$, lane 1) was annealed to a $5^{\prime}$ end-labelled probe and subjected to S1 nuclease digestion. Panel $\mathbf{C}$ shows the PstI 5 ' end probe used for the S1 nuclease analysis. Exon 1 of the mouse Thy-1.2 gene is represented by an open box. $\mathrm{P}$ indicates the position of the input primer or the input probe. $\mathrm{M}$ indicates the pBR322 x Hinfl marker fragments. Arrows indicate the major extended product or protected fragment in the respective experiments.

gene has been determined. The gene consists of four exons, which correspond approximately to the functional domains of the protein. The transcription unit of the mouse gene spans $6 \mathrm{~kb}$ of genomic DNA of which the coding and non-coding regions total $1700 \mathrm{bp}$ after processing. This is consistent with a size of 1850 nucleotides determined for the Thy-1 mRNA on Northern blots (Figure 5), assuming a poly(A) tail of $\sim 150$ nucleotides (Sawiki et al., 1977). Using primer extension and S1 nuclease mapping assays, we have defined the borders of the transcriptional unit. The Thy-1 mRNA has multiple sites of transcription initiation at the $5^{\prime}$ end, and a single polyadenylation site at the $3^{\prime}$ end of the gene. This last finding contradicts Moriuchi et al. (1983), indicating a cloning artefact rather than their suggestion that the Thy-1 gene is subject to differential polyadenylation. 


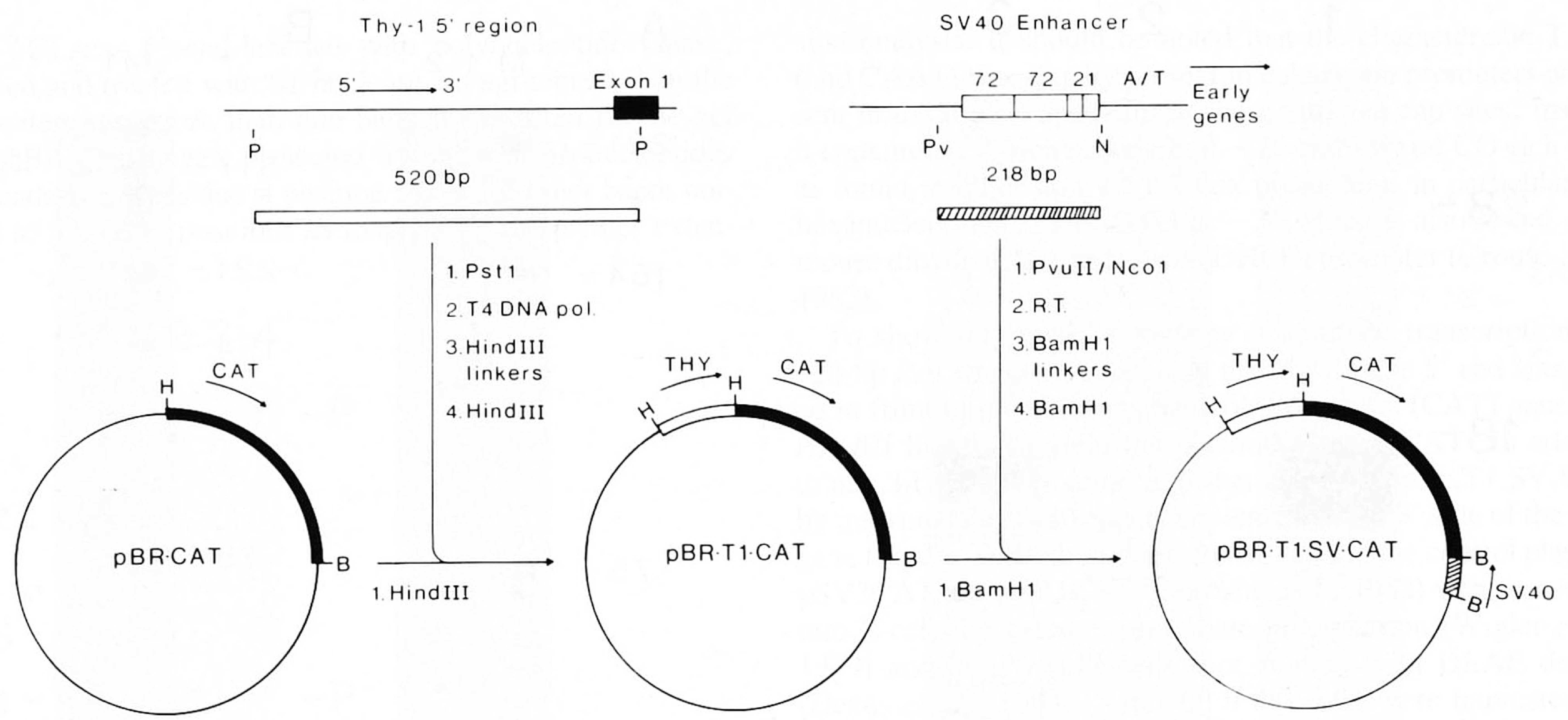

Fig. 7. Construction of CAT expression vectors containing the $5^{\prime}$ region of the Thy- 1 gene and the SV40 enhancer. pBR CAT was constructed as described (Walker et al., 1983). The 520-bp PstI fragment containing the Thy-1 promoter was obtained from pBSTI. The $P v u I I / N c o$ I restriction fragment containing the SV40 enhancer was obtained from pSV2-CAT (Gorman et al., 1982). Arrows indicate the direction of transcription. Enzymes used: H, HindIII; B, BamHI; P, PstI; N, NcoI; Pv, PvuII; RT, reverse transcriptase.

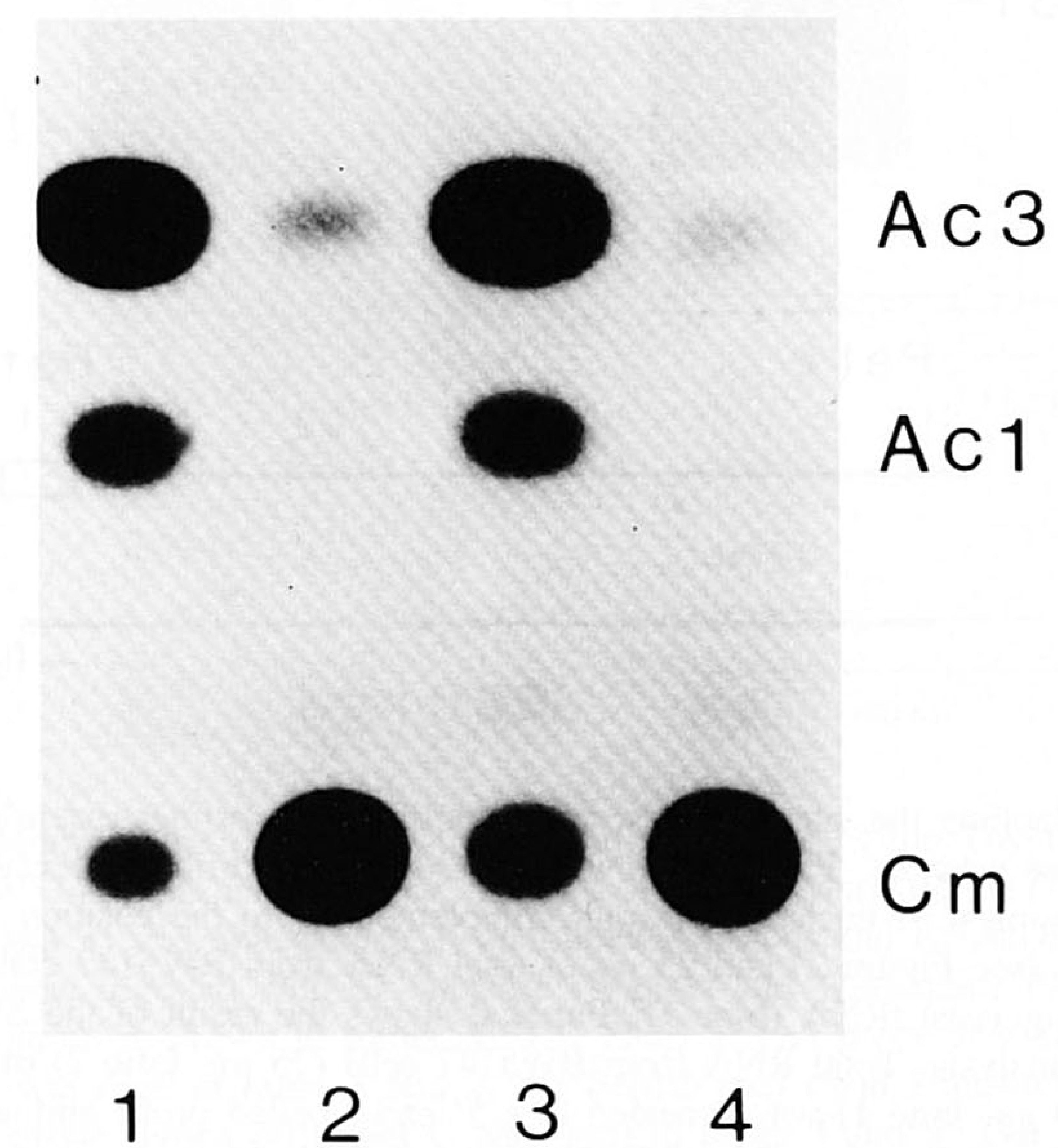

Fig. 8. Assay of CAT activity in mouse L-cells transfected with CAT expression vectors. Cells were transfected with $10 \mu \mathrm{g}$ of DNA and assayed $60 \mathrm{~h}$ later for CAT activity. Lane 1, pSV2-CAT; lane 2, pSV1-CAT; lane 3, pBRT1-SV-CAT; lane 4, pBRT1-CAT. CM, chloramphenicol; Acl, 1-acetylchloramphenicol; Ac3, 3-acetyl-chloramphenicol.

Although the physiological role of Thy- 1 is still not clear, the analysis of its exon/intron structure fully confirms its homology with the Ig superfamily of proteins (Williams and Gagnon, 1982), the different functional domains of the protein are encoded on separate exons with the exception of the $5^{\prime}$ leader sequence of the gene which is divided over two exons. This property is shared by $\beta 2$-microglobulin, the only other published protein in the Ig superfamily that contains a single extra cellular domain. Thy-1 might therefore be more closely related to $\beta 2$-microglobulin than was suggested by Hood et al. (1985). Comparison of the Thy-1 genes from different mouse strains shows that the Thy-1.1 gene is more similar to the C57BL/6 Thy-1.2 (Seki et al., 1985) than is the Balb/c Thy-1.2 gene. At present we do not know whether this indicates a high degree of polymorphism of the Thy-1 gene or whether this represent a very limited number of variant genes propagated by inbreeding.

The mouse Thy-1 glycoprotein is expressed in a developmental stage- and tissue-specific manner, its highest levels being found at the cell surface of thymocytes and brain cells. Recently, reintroduction of a cloned Thy-1.2 gene into different cell lines showed that the resulting transformants express up to 50-fold more cell surface Thy-1.2 antigen when the cell lines transformed already expressed the Thy-1 antigen at its cell surface (Evans et al., 1984). Although no data on the levels of the Thy-1 mRNA were presented, these results suggest that the Thy-1 gene might be subject to cell-specific transcriptional control. Thus, analysis of the mouse Thy-1 gene structure is a crucial first step toward studying the mechanisms which regulates its expression. Interestingly, sequencing analysis reveals that the Thy- 1 promoter is unusual. The immediate $5^{\prime}$-flanking region of the putative cap site contains a relatively high $\mathrm{G} / \mathrm{C}$ content $(68 \%)$ and lacks the conserved TATA and CAAT boxes normally found in the majority of eukaryotic promoters (Corden et al., 1980). Few promoters which share these unusual characteristics have been described. They include viral promoters (Baker et al., 1979; Baker and Ziff, 1981) the human $\beta$-tubulin gene (Lee et al., 1983) as well as the genes encoding different 'housekeeping' enzymes (Crouse et al., 1982; Melton et al., 1984; Reynolds et al., 1984; Valerio et al., 1985). For most of these genes, the absence of a conserved TATA box produces mRNAs whose $5^{\prime}$ termini are heterogeneous, a phenomenon also observed for the Thy-1 mRNA. Short regions of homology have been observed between certain of these promoters, especially with the sequence CCGCCC present in the SV40 early transcriptional unit (Reynolds et al., 1984; Melton et al., 1984). However, only one strong region of homology could be detected when the Thy-1 promoter was compared with the above-mentioned genes at position -34 with the corresponding region of the mouse DHFR gene (Crouse et al., 1982). This makes the Thy-1 promoter unique and deletion mutant studies are in progress to identify sequences responsible for the correct initiation of the Thy-1 mRNA per se. 


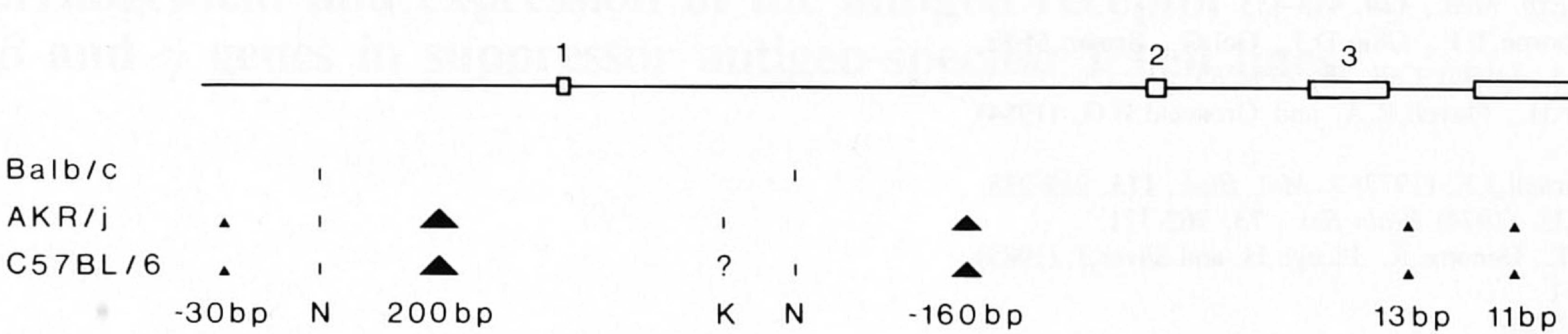

Fig. 9. Comparison of the Thy-1 locus in different mouse strains. Open boxes indicate exons. Different size triangles show different size DNA insertions and (|) indicates the presence of a restriction site. N, NcoI; K, KpnI.

In addition, preliminary results suggest that more than just the $5^{\prime}$ end of the gene is required to obtain tissue-specific expression (Giguere, unpublished).

Previous studies (Stern et al., 1975) have shown that the Thy-1 antigen can be detected on differentiated embryonal carcinoma cells. This observation is confirmed for F9 EC cells at the level of Thy-1 mRNA (Figure 5). At present we cannot distinguish between the two possible mechanisms for the increase of the mRNA; either stabilization of Thy-1 mRNA (which was rapidly turned over before differentiation) or alternatively, a transcriptional activation. Either way, since F9 EC cells can be transformed by DNA-mediated gene transfer and give rise to regulated expression of exogenous genes (Rosenthal et al., 1984), this should provide an interesting system to study the early expression of Thy-1.

\section{Materials and methods}

\section{Isolation of Thy-1 genomic clones}

The murine Thy-1.2 gene was isolated from a cosmid pTCF library of a Balb/c WEHI 3B (Warner et al., 1969) cell line DNA as described by Grosveld et al. (1982b). The library was screened with a Thy-1.2 cDNA insert as a probe (Moriuchi et al., 1983). One positive clone was isolated and mapped by standard double restriction enzyme digests. The murine Thy-1.1 gene was cloned from AKR DNA which was enriched for the presence of the 8.2-kb Thy-1 EcoRI fragment by preparative gel electrophoresis. The purified material was cloned into $\lambda 641$ (Murray et al., 1977) to yield a partial library of $10^{5}$ recombinant phage plaques. Twelve positives were picked after hybridization with a $B s t$ EIIApaI Thy-1.2 third exon probe. The 8.2-kb Thy-1 containing EcoRI fragments were isolated by gel electrophoresis and recloned in the expression vector pBSV (Grosveld et al., 1982a) by standard procedures.

DNA-mediated transfection and CAT assay

The recombinant DNA construct, either with pBSV or the CAT plasmids were introduced into eukaryotic cells by calcium phosphate co-precipitation (Wigler et al., 1979) or by DEAE-dextran as described by Deans et al. (1984). After transfection of HeLa cells with the pBST1 (Thy-1 pBSV) the cells were harvested after 2 days in culture ( $\alpha$ MEM 10\% NBCS) and analyzed for the presence of the Thy- 1 antigen on the cell surface with a monoclonal anti-Thy- 1 antibody by standard procedures. After transfection with the CAT gene constructs the mouse L-cells were grown for $72 \mathrm{~h}$ in $\alpha$ MEM $10 \%$ NBCS and prepared for CAT assays as described by Gorman et al. (1982).

\section{Analysis of the Thy-1.2 mRNA}

Total RNA was isolated from cells in culture by the guanidium/caesium chloride method (Glisin et al., 1974; Ullrich et al., 1977). Northern blot analysis was performed as described by Thomas (1983). S1 nuclease analysis was performed as described (Berk and Sharp, 1977; Weaver and Weissman, 1979) using probes end labelled by $\mathrm{T} 4$ polynucleotide kinase or reverse transcriptase to a specific activity $>10^{7}$ c.p.m. $/ \mu \mathrm{g}$.

For primer extension analysis, a 474-bp XbaI/BstEII restriction fragment was purified on agarose gel, cut with Hinfl, end-labelled by T4 polynucleotide kinase, recut by $H$ haI and repurified on an $8 \%$ polyacrylamide- $7 \mathrm{M}$ urea gel. The singlestranded 31 nucleotide long Hinfl-HhaI primer was eluted from the gel and its purity confirmed by sequencing. We suspended $25 \mu \mathrm{g}$ of total RNA and $\sim 0.2$ pmol of ${ }^{32} \mathrm{P}$-labelled primer in $10 \mu \mathrm{l}$ of buffer containing $400 \mathrm{mM} \mathrm{NaCl}, 10 \mathrm{mM}$ Pipes pH 6.4. The mixture was sealed in a glass capillary, denatured at $90^{\circ} \mathrm{C}$ for $2 \mathrm{~min}$, and allowed to hybridize overnight at $50^{\circ} \mathrm{C}$. To initiate primer extension, the glass capillary was broken and its contents delivered into reactive mix- ture containing $50 \mathrm{mM}$ Tris $\mathrm{HCl} \mathrm{pH} 8.2,10 \mathrm{mM}$ DTT, $6 \mathrm{mM} \mathrm{MgCl}, 2,5 \mathrm{mM}$ of each dNTP, $2.5 \mu \mathrm{g}$ actinomycin D and 10 units of reverse transcriptase. The reaction was incubated at $41^{\circ} \mathrm{C}$ for $1 \mathrm{~h}$ and the extended products analyzed by electrophoresis on $8 \%$ polyacrylamide- $7 \mathrm{M}$ urea gel followed by autoradiography.

\section{Acknowledgements}

We are grateful to Roger James and Arnon Rosenthal for their assistance and to Cora O'Carroll for her speedy preparation of the manuscript. V.G. was supported by the Medical Research Council of Canada. K-I.I. was supported by a visiting I.C.R.F. fellowship. The work was supported by the Medical Research Council UK.

\section{References}

Baker,C.C. and Ziff,E.B. (1981) J. Mol. Biol., 149, 189-221.

Baker,C.C., Heresse,J., Courtois,G., Galibert,F. and Ziff,E. (1979) Cell, 18, 569-580.

Banerji,J., Rusconi,S. and Schafner,W. (1981) Cell, 27, 299-303.

Bash,R. and Berman,S. (1982) Eur. J. Immunol., 12, 359-364.

Benoist,C., O'Hare,K., Breathnach, R. and Chambon,P. (1980) Nucleic Acids Rs., 8, 127-142.

Berk,A. and Sharp,P. (1977) Cell, 12, 721-732.

Blankenhorn,E. and Douglas, T. (1972) J. Hered., 63, 259-263.

Campbell,D.G., Gagnon,J., Reid,K.B.M. and Williams,A.F. (1981) Biochem. J., $195,1-30$.

Clark,M.J., Gagnon,J., Williams,A.F. and Barclay,A.N. (1985) EMBO J., 4, 113-118.

Cohen,F.E., Novotny,J., Sternberg,M.J.E., Campbell,D.G. and Williams,A.F. (1981) Biochem. J., 195, 31-40.

Corden,J., Wasylyk,A., Buchwalder,A., Sassone-Corsi,P., Kedinger,C. and Chambon,P. (1980) Science (Wash.), 209, 1406-1414.

Cotmore,S.F., Crowhurst,S.A. and Waterfield,M.D. (1981) Eur. J. Immunol., 11, 597-603.

Crouse,G.F., Simonsen,C.C., McEwan,R.N. and Schimke,R.T. (1982) J. Biol. Chem., 257, 7887-7897.

Deans,R.J., Denis,K.A., Taylor,A. and Wall,R. (1984) Proc. Natl. Acad. Sci. USA, 81, 1292-1296.

Evans,G.A., Ingraham,H.A., Lervis,K., Cunningham,K., Seki,T., Moriuchi,T., Chang,H.C., Silver,J. and Hyman,R. (1984) Proc. Natl. Acad. Sci. USA, 81, 5532-5534.

Glisin,V., Crkvenjakov,R. and Byus,C. (1974) Biochemistry (Wash.), 13, 2633-2637.

Goldsby,R., Osborne,B., Simpson,E. and Herzenberg,R. (1977) Nature, 267, 707-708.

Gorman,C., Moffat,L. and Howard,B. (1982) Mol. Cell. Biol., 2, 1044-1051.

Grosveld,G.C., deBoer,E., Shewmaker,C.K. and Flavell,R.A. (1982a) Nature, 295, 120-126.

Grosveld,F.G., Lund,T., Mellor,A.L. and Flavell,R.A. (1982b) Nucleic Acids Res., 10, 6715-6732.

Hogan,B.L., Taylor,A. and Adamson,E. (1981) Nature, 291, 235-237.

Hood,L., Kronenberg,M. and Hunkapiller,T. (1985) Cell, 40, 225-229.

Lake,P. (1979) Eur. J. Immunol., 9, 875-879.

Lee,M.G.-S., Lewis,S.A., Wilde,C.D. and Cowan,N.J. (1983) Cell, 33, 477-487.

McNicholas,J., Steinmetz,M., Hunkapiller,T., Jones,P. and Hood,L. (1982) Science (Wash.), 218, 2339-1232.

Melton,D.W., Konecki,D.S., Brennand,J. and Caskey,C.T. (1984) Proc. Natl. Acad. Sci. USA, 81, 2147-2151.

Moriuchi,T., Chang,H.-C., Denome,R. and Silver,J. (1983) Nature, 301, 80-82. Mostov,K.E., Friedlander,M. and Blobel,G. (1984) Nature, 308, 37-43.

Murray,N.E., Brammar,W.J. and Murray,K. (1977) Mol. Gen. Genet., 150, 53-61. 
Owen,J. and Raff,M. (1970) J. Exp. Med., 132, 1216-1232.

Reif,A. and Allen,J. (1964) J. Exp. Med., 120, 413-433.

Reynolds,G.A., Basu,S.K., Osborne,T.F., Chin,D.J., Gel,G., Brown,M.S., Goldstein,J.L. and Luskey,K.L. (1984) Cell, 38, 275-285.

Rosenthal,A., Wright,S., Cedar,H., Flavell,R.A. and Grosveld,F.G. (1984) Nature, 310, 415-418.

Sawiki,S.G., Jelinek,W. and Darnell,J.E. (1977) J. Mol. Biol., 113, 219-235.

Schachner,M. and Hämmerling,U. (1974) Brain Res., 73, 362-371.

Seki,T., Chang,J.-C., Meriuchi,T., Denome,R., Ploegh,H. and Silver,J. (1985) Science (Wash.), 227, 649-651.

Sharp,P.A. (1981) Cell, 23, 643-646.

Stern,P.L., Martin,G.R. and Evans,M.J. (1975) Cell, 6, 445-465.

Thomas,P.S. (1983) Methods Enzymol., 100, 255-265.

Ullrich,A., Shine,J., Chirgwin,J., Pictet,R., Tischer,E., Rutter,W.J. and Goodman,H.M. (1977) Science (Wash.), 196, 1313-1319.

Valerio,D., Duyvesteyn,M.G.C., Dekker,B..M., Weeda,G., Berkvens,T.M., van der Boom,L., van Ormonde,H. and van der Eb,A.J. (1985) EMBO J., 4, 437-443.

Walker,M.D., Edlund,T., Boulet,A.M. and Rutter,W.J. (1983) Nature, 306, 557-561.

Warner,N.L., Moore,M.A.S. and Metcalf,D. (1969) J. Natl. Cancer Inst., 43, 963-982.

Weaver,R. and Weissman,S. (1979) Nucleic Acids Res., 7, 1175-1193.

Wigler,M., Sweet,R., Sim,G.K., Wld,B., Pellicer,A., Lacy,E., Maniatis,T., Silverstein,S. and Axel,R. (1979) Cell, 16, 777-785

Williams,A.F. and Gagnon,J. (1982) Science (Wash.), 216, 696-703.

Received on 10 April 1985; revised on 29 May 1985 